\title{
Transcranial Ultrasound: Efficient Screening Tool for Detection of Hypoxic Brain Injury in Neonates - Study of 50 Patients
}

\author{
Pinkesh Herma ${ }^{1}$, Jagruti Kalola ${ }^{2}$, Mahak Sood ${ }^{3}$, Anjana Trivedi ${ }^{4}$ \\ ${ }^{1} 3^{\text {rd }}$ Year Resident, ${ }^{2}$ Associate Professor, ${ }^{3}$ Assistant Professor, ${ }^{4}$ Head of Department, Department of Radio-Diagnosis and \\ Imaging, P.D.U. Government Medical College and Civil Hospital, Rajkot, Gujarat, India.
}

Corresponding author: Dr. Pinkesh Herma, 403, Ramvatika Apartment, Marutinagar Street No 3, Airport Road, Rajkot -360001 , India

DOI: 10.21276/ijcmsr.2018.3.3.20

How to cite this article: Pinkesh Herma, Jagruti Kalola, Mahak Sood, Anjana Trivedi. Transcranial ultrasound: efficient screening tool for detection of hypoxic brain injury in neonates - study of 50 patients. International Journal of Contemporary Medicine Surgery and Radiology. 2018;3(3):C93-C95.

\section{A B S T R A C T}

Introduction: Hypoxic-ischemic encephalopathy (HIE) is a major contributor to neonatal neurological morbidity and mortality. Early evaluation and detection plays important role in prognosis of hypoxic-ischemic encephalopathy (HIE). Transcranial ultrasound and MRI are standard imaging modality for the diagnosis.

Material and Methods: Total 50 neonates with history of birth asphyxia, delayed cry or convulsion with unknown etiology evaluated by bedside transcranial ultrasound. Each patient would undergo further imaging and clinical examination for confirmation of the diagnosis.

Results: Out of 50 patients 41 patients were positively identified by transcranial ultrasound which further confirmed clinically and radiologically.

Conclusions: Bedside transcranial ultrasound appears to be very sensitive and promising in early detection and management of the hypoxic brain injury. In our study it proves to be valuable screening tool for hypoxic brain injury.

Key words: Transcranial Ultrasound, Hypoxic Brain Injury, PVL (Periventricular Leucomalacia)

\section{INTRODUCTION}

Hypoxic-ischemic encephalopathy (HIE) is most common clinically encountered problem in neonatal ICU. hypoxicischemic encephalopathy (HIE) is significantly associated with neurological demise and cerebral palsy in neonates. ${ }^{1}$ Even in developed countries, neonates having moderate to severe hypoxic-ischemic encephalopathy death or moderate to severe disability occurs in $53-61 \%$ of infants. ${ }^{2}$ In hypoxicischemic encephalopathy, neonates brain shows high levels of cerebral blood flow measured at 12-24 hours of life have been associated with more severe brain injury. In infant's asphyxia considered to be most common risk factor for brain injury however severity of injury mainly depends on duration of asphyxia and brain maturity. ${ }^{3,4}$ Asphyxia affects brain of full term neonates differently than preterm neonates. In neonates with asphyxia brain monitoring and functional assessment done by MRI and electroencephalography however now a day's ultrasound provides early sensitive diagnostic tools with its advantage like portability and lack of ionizing radiation. With help of recent advancement high resolution transcranial ultrasound has provided reliable and comprehensive information about extent and severity of brain injury in asphyxiated neonates. ${ }^{5}$

Apart from hypoxic brain injury transcranial ultrasound useful in detection of intracranial and intraventricular hemorrhage, congenital malformation like post fossa malformation
(Dendy walker complex), schizencephaly, arachnoid cyst and intraventricular tumours.

Aim of our study was to detect hypoxic brain injury in neonates and grading of hypoxic insult according to involvement of brain parenchyma.

\section{MATERIAL AND METHODS}

This retrospective study was conducted in the department of radio-diagnosis and imaging, P.D.U. Medical College and Civil hospital, Rajkot, Gujarat over a period of six months from December 2017 to May 2018. Total 50 patients were included in study who was undergone bedside transcranial ultrasound. Transcranial ultrasound was done using Philips ultrasound system with high frequency probe.

Study location: This was a tertiary care teaching hospital based study done in Department of radio-diagnosis, at P.D.U medical college and civil Hospital, Rajkot, Gujarat.

\section{Patient selection}

Inclusion criteria

Neonates with birth asphyxia

Preterm neonates

Neonates with convulsion of unknown etiology

\section{Exclusion criteria}

Age more than 1 year

Full term infants with normal cry 


\begin{tabular}{|l|l|l|l|}
\hline Transcranial ultrasonography & $\begin{array}{l}\text { Total Number of } \\
\text { patients }\end{array}$ & $\begin{array}{l}\text { Total Full term } \\
\text { Neonates }\end{array}$ & Total preterm Neonates \\
\hline Hypoxic-ischemic encephalopathy & $41(82 \%)$ & $27(65 \%)$ & $14(35 \%)$ \\
\hline Normal ultrasound & $9(18 \%)$ & $6(67 \%)$ & $3(33 \%)$ \\
\hline \multicolumn{2}{|r|}{ Table-1: Incidence (percentage) of Hypoxic-ischemic encephalopathy in pediatric patients } \\
\hline
\end{tabular}

\begin{tabular}{|l|l|l|}
\hline $\begin{array}{l}\text { Grade of periventricular } \\
\text { leucomalacia (PVL) }\end{array}$ & $\begin{array}{l}\text { Full term } \\
\text { neonates }\end{array}$ & $\begin{array}{l}\text { Preterm } \\
\text { neonates }\end{array}$ \\
\hline Grade 1 & 2 & 0 \\
\hline Grade 2 & 6 & 1 \\
\hline Grade 3 & 9 & 5 \\
\hline Grade 4 & 10 & 8 \\
\hline Total & 27 & 14 \\
\hline \multicolumn{2}{|l|}{ Table-2: Incidence of PVL in full term and preterm neonates } \\
\hline
\end{tabular}

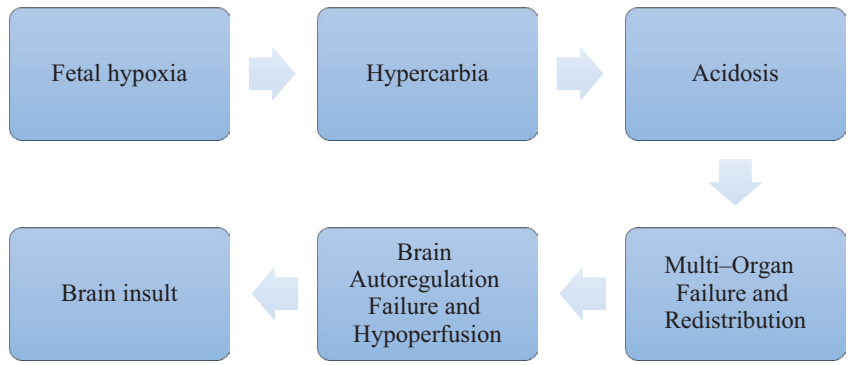

Figure-1: Algorithm showing step by step pathophysiology of hypoxic brain injury

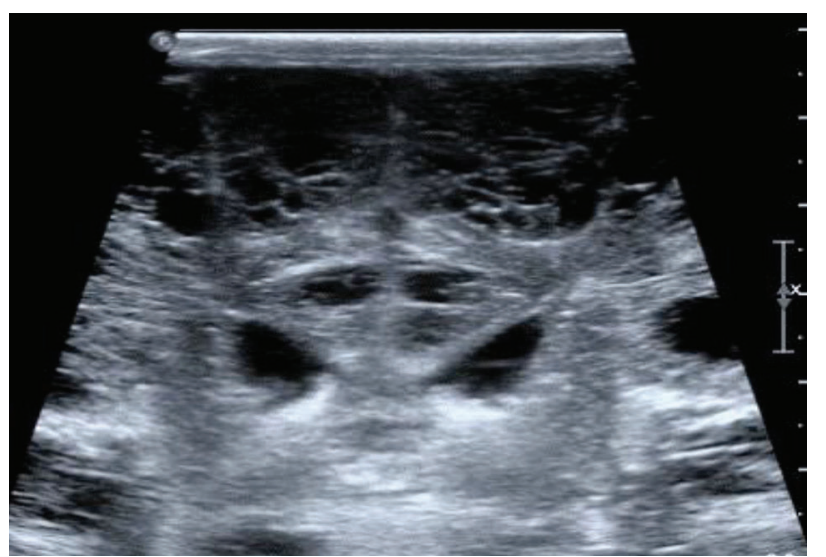

Figure-2: coronal section using high frequency linear probe showing extensive encephalomalacia and also involvement of cerebral cortex. (grade 4 PVL)

\section{DISCUSSION}

Out of 50 patients 41 was correctly identified having brain injury secondary to hypoxia which was confirmed clinically and further evaluation on magnetic resonance imaging. 9 patients appear to be normal on ultrasound in which further magnetic resonance imaging shows brain injury in 5 patients and rest of the patients have normal study. Out of 50 patients 41 patient diagnosed with hypoxic injury among which 27 (65\%) were full term and 14 (35\%) were preterm (table 1).

According to our study transcranial ultrasound shows $82 \%$ of sensitivity in diagnosis of hypoxic-ischemic encephalopathy (HIE) with $11 \%$ of false negative rate.

Sensitivity for detection of grade 3 and 4 periventricular leucomalacia by transcranial ultrasound is $50-60 \%$ as compared to grade 1 and 2 periventricular leucomalacia. In our study we calculated incidence of PVL in full and preterm neonates. Results shows incidence of grade 3-4 PVL is higher than grade 1-2 PVL (table 2).

Study shows preterm infants are more commonly affected and most susceptible to hypoxic-ischemic encephalopathy (HIE). Severity of injury also depends on brain maturity. Premature brain shows sever brain insult then mature brain. In our case $90 \%$ of preterm have high grade periventricular leucomalacia as compared to full term $70 \%$.
Birth asphyxia in neonates and preterm mainly affects deep gray matter like putamen, ventrolateral thalami, hippocampi, brainstem, and lateral

geniculate nuclei. These areas of the brain contain the highest concentrations of NMDA receptors and have high metabolic demands (because of ongoing myelination) therefore, the most susceptible to neonatal hypoxic-ischemic encephalopathy (HIE). ${ }^{6}$

Donna Ferriero et al suggested that mortality and morbidity from neonatal brain injury is significantly high and associated with long term effects in form of stroke, status epilepticus and cerebral palsy. ${ }^{3}$

Transcranial ultrasound in early life first 7 days have proven less sensitive for detection of changes of hypoxic-ischemic encephalopathy (HIE). After first week changes are more evident. Early transcranial ultrasound findings include increase cortical echogenicity, obliteration of CSF spaces because of edema, increase thalamic echogenicity, persistent periventricular echogenicity. Thalamic involvement associated with poor outcome and more severe injury. Late findings include cystic changes involving cortex and subcortical area, encephalomalcia, ex vacuo ventricular dilatation, prominent 
extra-axial CSF spaces, rarely porencephalic cyst formation. ${ }^{7}$ Periventricular leucomalacia is term used for white matter disease occurs in hypoxic-ischemic encephalopathy (HIE).In premature this white matter zone is a watershed zone between deep and superficial vessels so always prone to ischaemia in case of birth asphyxia. Periventricular leucomalacia occurs in form of periventricular echogenic foci. PVL occurs most commonly in premature infants born at less than 33 week's gestation (38\% PVL) and less than $1500 \mathrm{~g}$ birth weight (45\% PVL). ${ }^{8}$

Sie et al suggested grading for periventricular leucomalacia a form of neonatal hypoxic injury. they also suggested simultaneous use of transcranial ultrasound and MRI of brain for detection of acute periventricular leucomalacia. ${ }^{6}$ Transcranial ultrasound is recommended as screening tool in each and every cases (figure-2).

\section{Grading of periventricular leucomalacia}

Grade 1: Increase periventricular echogenicity persisting more than 7 days

Grade 2: Developing small periventricular cyst

Grade 3: Developing extensive periventricular cyst extending in occipital, fronto-parietal lobe

Grade 4: Extensive subcortical cyst formation

Transcranial ultrasound also provides valuable information by Doppler study of anterior and middle cerebral artery. Hypoperfusion can be detected very early with highest sensitivity by transcranial Doppler study. Study have shown Low resistive index is significantly associated with poor outcome. ${ }^{9}$

Magnetic resonance imaging is most sensitive and standard imaging modality for hypoxic-ischemic encephalopathy (HIE). Recent advance with perfusion study, MR spectroscopy and diffusion weighted imaging have made possible to detect hypoxic insult earlier than any modality. Apart from gliosis, encephalomalacia, hemorrhage Magnetic resonance imaging can detect abnormalities like heterotopia, focal cortical dysplasia which cannot be detected by transcranial ultrasound. MR imaging have low false negative rate as compared to transcranial ultrasound.

Advantages of transcranial ultrasound includes cheap, easy availability, good learning curve as compared to MRI, can be done on bed side even if neonates are not stable, and sensitive for detection of hypoxic brain damage. MRI is costly, require stable patient and cannot be done on bedside limits its utility as handy bedside screening tool in spite of high sensitivity and specificity for detection of hypoxic-ischemic encephalopathy (HIE).

\section{CONCLUSION}

Transcranial ultrasound proved to be very useful bedside screening tool for detection of hypoxic-ischemic encephalopathy (HIE) with sensitivity of $82 \%$ in our study. Although MRI is a gold standard practice of initial screening of high risk neonates by means of transcranial ultrasound in early life is recommended because it provides useful information regarding extent and severity of hypoxicischemic encephalopathy (HIE).

\section{REFERENCES}

1. Shankaran S, Laptook AR, Ehrenkranz RA, et al. Whole-body hypothermia for neonates with hypoxicischemic encephalopathy. N Engl J Med 2005; 353(1):1574-1584

2. Kurinczuk JJ, White-Koning M, Badawi N. Epidemiology of neonatal encephalopathy and hypoxicischaemic encephalopathy. Early Hum Dev. 2010;86(2):329-38.

3. Ferriero DM. Neonatal brain injury. N Engl J Med. 2004;351(4):1985-95.

4. Johnston MV, Trescher WH, Ishida A, Nakajima W. Neurobiology of hypoxic-ischemic injury in the developing brain. Pediatr Res. 2001;49(6):735-41.

5. Barkovich AJ, editor. Pediatric Neuroimaging. 3rd ed. New York: Lippincott Williams and Wilkins; 2000. pp. 162-208.

6. Sie LT, van der Knaap MS, van Wezel-Meijler G, Taets van Amerongen AH, Lafeber HN, Valk J. Early MR features of hypoxic-ischemic brain injury in neonates with periventricular densities on sonograms. AJNR Am J Neuroradiol. 2000;21(3):852-61.

7. Shalak L, Perlman JM. Hypoxic-ischemic brain injury in the term infant: current concepts. Early Hum Dev 2004;80(2):125-141.

8. Miller SP, Ramaswamy V, Michelson D, et al. Patterns of brain injury in term neonatal encephalopathy. J Pediatr 2005; 146(5):453-460

9. Cassia GS, Faingold R, Bernard C, Sant'Anna GM. Neonatal Hypoxic-Ischemic Injury. Sonography and Dynamic Color Doppler Sonography Perfusion of the Brain and Abdomen With Pathologic Correlation. AJR Am J Roentgenol. 2012;199(6):W743-52

\section{Source of Support: Nil; Conflict of Interest: None}

Submitted: 21-06-2018; Accepted: 12-08-2018; Published online: 19-08-2018 